\title{
Muertes invisibles entre jóvenes en la zona sur de la Ciudad de Buenos Aires: reconstrucciones biográficas e itinerarios de la experiencia de familiares y amigos
}

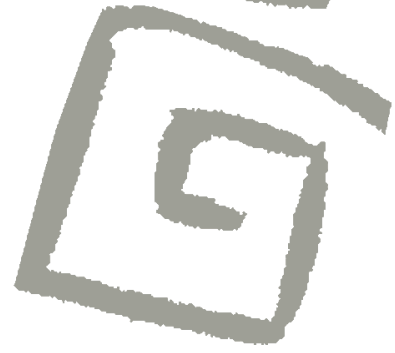

\author{
Invisible deaths among youth in the south of Buenos \\ Aires: biographical reconstructions and itineraries of the \\ experience of families and friends
}

Alejandro Marcelo Villa ${ }^{1}$

'Licenciado en Psicología. Especialista en Ciencias Sociales y Salud. Investigador asociado, Consejo de Investigación en Salud, Ministerio de Salud, Gobierno de la Ciudad Autónoma de Buenos Aires. Buenos Aires, Argentina. $\triangle$ iD
RESUMEN Varias fuentes de datos secundarios y algunos estudios previos destacan la magnitud de los homicidios entre jóvenes en las poblaciones marginalizadas de la zona sur de la Ciudad de Buenos Aires. Con un enfoque que incorpora las perspectivas de la sociología de la individuación, la antropología de las moralidades y de los procesos psíquicos involucrados en la subjetivación, se realizó un estudio de casos en profundidad, entre los años 2014 y 2016. Se reconstruyó un conjunto de biografías de jóvenes muertos frente a otros jóvenes, con testimonios de familiares, y de jóvenes amigos, de pares muertos. El trabajo se propone profundizar en los diferentes contextos y condiciones de posibilidad de las experiencias de estos actores, en que el proceso de reconstrucción biográfica vinculado a las sociabilidades juveniles, junto al dolor y las vulnerabilidades, tras una muerte, podrían conducir a una crítica social de la naturalización de la violencia entre jóvenes, en la vida cotidiana de los barrios marginalizados.

PALABRAS CLAVES Jóvenes; Violencia; Muerte; Biografía; Argentina.

\begin{abstract}
Many sources of secondary data and some previous studies highlight the magnitude of homicides among young people in marginalized populations of the southern part of the City of Buenos Aires. With an approach that incorporates the perspectives of the sociology of individuation, the anthropology of moralities, and the psychic processes involved in subjectivation, an in-depth cases study was conducted between the years 2014 and 2016. A set of biographies of young people who died in front of other young people were reconstructed through the testimonies of families and friends of dead youth. The work aims to delve into the different contexts and conditions of possibility of the experiences of these actors, in which the process of biographical reconstruction linked to youth sociabilities, along with pain and vulnerabilities after a death, can lead to a social critique of the naturalization of the violence among young people in the everyday life of marginalized neighborhoods.
\end{abstract}

KEY WORDS Youths; Violence; Death; Biography; Argentina. 


\section{ANTECEDENTES Y PROBLEMA DE ESTUDIO}

La magnitud de la violencia juvenil en la región latinoamericana, así como la creciente presencia de las muertes de jóvenes por causas violentas, han sido destacadas por documentos regionales ${ }^{(1)}$, que Ilaman la atención sobre una serie de problemas: el debilitamiento de los mecanismos formales e informales de protección social de los jóvenes; un exacerbamiento de las diferencias generacionales; una sensación de exclusión social, caracterizada por distintas formas de violencia física y simbólica hacia ellos; y un aumento de los homicidios juveniles masculinos.

La región latinoamericana presenta las tasas más altas de muertes violentas del mundo ${ }^{(2)}$. Las víctimas de la violencia homicida en América Latina, son fundamentalmente varones jóvenes y pobres, y el homicidio se constituye en la primera causa de muerte en jóvenes en América Latina. En Argentina, se trata de una de cada cinco muertes ${ }^{(3)}$.

Algunos estudios se proponen vincular la violencia interpersonal y las muertes de jóvenes con un conjunto de fenómenos sociales que acontecen a nivel regional: las condiciones de desigualdad social junto al desempleo de los jóvenes; la pérdida de control de la familia sobre la socialización de las y los jóvenes, las expectativas de consumo y condiciones materiales desiguales, la organización territorial de las ciudades donde se concentra la pobreza con alta densidad poblacional, y los patrones de violencia masculina como mecanismo de género en tanto modo de obtención de prestigio social(3).

En la Argentina, son escasos los estudios específicos que caracterizan las condiciones psicosociales en que se produce la violencia interpersonal y la muerte entre jóvenes. La Corte Suprema de Justicia de la Nación ${ }^{(4)}$ realiza un seguimiento de los registros de homicidios dolosos en la Ciudad Autónoma de Buenos Aires (CABA), sobre la base de expedientes judiciales. En todos los homicidios interviene el registro del poder judicial del Estado. Para el año 2011, se contabilizaron
184 causas judiciales y 190 víctimas. Como en este seguimiento se investigan los hechos vinculados a los homicidios, una misma causa judicial puede incluir más de un homicidio.

Allí se destaca que se produce una concentración de los homicidios en las poblaciones urbanas más pauperizadas. Son las denominadas "villas de emergencia", casi en su totalidad localizadas en la zona sur de la ciudad (villa 21-24 y Núcleo Habitacional Transitorio Zavaleta, villa 1-11-14 y villa 15), a excepción de la villa 31-31 Bis, localizada en la zona norte. El $27 \%$ de las víctimas en toda la CABA tiene entre 18 y 25 años; de las cuales, el 36\% residía en "villas de emergencia". Se destacan allí las situaciones de violencia interpersonal entre grupos de jóvenes, en su mayoría en la "calle", y con armas de fuego y blancas. Los principales motivos de las muertes en toda la CABA corresponden a una sola categoría que agrupa "riña", "ajuste de cuentas" y "venganza", la cual representa un $39 \%$, mientras que el $15 \%$ corresponde exclusivamente a robos. En el análisis georeferenciado del mencionado estudio ${ }^{(4)}$ se muestra que el $73 \%$ de las muertes corresponden a la zona sur de la CABA (Comunas $1,4,7,8$ y 9). En cuanto a los motivos de muerte homicida en las "villas de emergencia", se destacan los vinculados a "riña", "ajuste de cuentas" y "venganza", con un $59 \%$, en contraste con el $39 \%$ del mismo motivo para el total de la CABA.

\section{La segregación social de los jóvenes y la violencia: la perspectiva del actor y las luchas por el reconocimiento}

En Argentina, durante las últimas décadas, los sectores populares urbanos más empobrecidos fueron socializados en espacios homogéneos que refuerzan o conducen al aislamiento social. Los cambios en los valores de la familia, el trabajo y la educación, como principales organizadores de la socialización, en conjunto con un proceso de segregación urbana, promovieron un proceso de exclusión social territorial, una fragmentación social y una vulnerabilidad social ${ }^{(5,6,7)}$. 
Al analizar las violencias de los jóvenes, Pegoraro $^{(8)}$ destaca que existen dos enfoques para explicar las "conductas desviadas de los jóvenes". Por un lado, "un enfoque estructural" que coloca a la violencia como consecuencia de las carencias materiales y la exclusión social. Por otro, un "enfoque cultural" que alude a la dificultad de los individuos de incorporar los valores sociales, frente a una "ausencia de una moral social respetuosa de la ley". Se pone énfasis en la noción de un "código cultural", en tanto un conjunto de valores y pensamientos homogéneos, que trasciende la acción misma de los actores sociales, y al cual la acción debería acomodarse o rendir cuentas. Asimismo, en la mayor parte de la bibliografía sociológica existente, se concibe a los jóvenes en términos negativos con respecto a las determinaciones sociales estructurales. Se busca adecuar las sociabilidades juveniles (familia, escuela, grupo de pares) y los procesos (de fragmentación y vulnerabilidad) a dichas determinaciones.

Para el presente estudio, se torna insuficiente analizar solo los efectos de la estructura de clase social y de las condiciones culturales homogéneas que determinarían las condiciones de socialización de los jóvenes. Por lo tanto, se propone un tercer enfoque que parta de la acción de los jóvenes y sus allegados en tanto actores sociales singulares, para lo cual se incorporan tres tipos de perspectivas: sociológicas, antropológicas, y de los procesos psíquicos que intervienen en la subjetivación.

Desde una perspectiva sociológica, es necesario pensar los desplazamientos y la circulación de los jóvenes por las distintas geografías y el modo de transitar los espacios de sociabilidad ${ }^{(9)}$; como también caracterizar los lazos sociales de los jóvenes de barrios marginalizados, en los que la violencia, el delito y la ilegalidad serían la expresión de un descontento juvenil, según la percepción de injusticias cotidianas ${ }^{(10)}$. Se destacan, además, los procesos de luchas por el reconocimiento identitario juvenil, frente a una "fragmentación" de los lazos sociales de los jóvenes con sus vecinos y con sus mismos pares, $y$ a una "degradación de las pautas socioculturales compartidas"(6). Los jóvenes pueden accionar una "demanda de respeto" frente a sus mismos pares y sus vecinos, mediante el ejercicio de la violencia, en un contexto de humillación, exclusión y falta de opciones sociales que puedan configurar una identidad valorada. Esta demanda puede concebirse como una capacidad intersubjetiva de reconocimiento igualitario o contrariamente una "demanda pura" de un sujeto que se impone unilateralmente por la fuerza sobre otro, estableciendo una desigualdad jerárquica ${ }^{(11,12)}$. La disputa de un territorio entre diferentes grupos de jóvenes, mediante la violencia y la muerte, responde a una materialización de la construcción de un sentido de otredad, en un contexto de relativa homogeneidad de subculturas juveniles ${ }^{(13)}$. Se trata de la restricción de territorios practicadas por grupos juveniles, en una oposición identitaria entre un "nosotros" y "ellos", en la cual se produce una segmentación de las sociabilidades juveniles entre "respeto" y "anti-respeto"(11).

Siguiendo las perspectivas de la antropología de las moralidades ${ }^{(14)}$, se puede entender que la muerte, como resultado de una disputa social entre grupos juveniles, pone en cuestión el pensamiento de los actores involucrados en dicha situación específica. La violencia y la muerte instituyen una ruptura en los modos de concebir al mundo social, a los otros y a la propia persona. Ello ocurre en una tensión entre la mirada comunitaria que juzga los comportamientos juveniles con valores y pensamientos sociales, y la mirada que adoptan los propios actores allegados al muerto. En el lugar de lo que se ha pensado como "código cultural", es necesario visibilizar la existencia de una heterogeneidad de valores que se encuentran en tensión y disputa por el sentido de la acción ${ }^{(15)}$. También, se trata de mecanismos de distribución de recursos cognitivos y morales que realiza el actor; los que entran en disputa con relación a quiénes deben ser los destinatarios ${ }^{(16)}$.

Una tercera perspectiva, psíquica y de subjetivación, reúne un conjunto de campos de estudios para vincular la reconstrucción de biografías con los espacios de sociabilidad 
transitados, el dolor que provocan las muertes y los procesos de vulnerabilidades que se desencadenan en los actores cercanos a las muertes $^{(17)}$. Esto comprende un proceso de desintegración del yo, que inaugura procesos de transmisión psíquica en el acontecer biográfico, que remiten a las posibilidades de una "inscripción psíquica" e "histórica" de la violencia y la muerte ${ }^{(17,18)}$.

\section{La vulnerabilidad como proceso y la experiencia de los actores tras una muerte}

Tomando distancia de la categoría de riesgo y de la vulnerabilidad concebida como una situación específica en el tiempo, se concibe aquí la vulnerabilidad como resultado de un proceso histórico, que comprende tres dimensiones que se articulan entre sí(19). En primer lugar, las trayectorias personales de los actores; en segundo lugar, los vínculos e interacciones, que pueden hacer que una trayectoria personal se transforme en una trayectoria social; $y$, en tercer lugar, los contextos socioinstitucionales, en los que se producen las trayectorias personales y sociales, y que pueden suministrar o negar el acceso a determinados recursos materiales y simbólicos.

Se torna necesario vincular este concepto de vulnerabilidad a la caracterización de la experiencia que enfrentan los actores allegados al joven muerto en estas situaciones. Para la sociología de la individuación de François Dubet ${ }^{(20)}$, la experiencia social parte de la acción del actor y no de un análisis de la estructura social. Al actor se le impone el trabajo de resolver cognitivamente "la paradoja de la doble afirmación del influjo de lo social y de la autonomía del actor". Se trata de dar cuenta de los modos en que este puede metabolizar lo social y producirlo, mediante una multiplicidad de lógicas de la acción. Sin embargo, en lugar de dar privilegio exclusivo a la acción racional y la actividad cognitiva del actor, el foco de su experiencia es dar sentido a la experiencia de vivir juntos. Ello supone objetivos afectivos, identitarios y simbólicos, mediante la constitución de "un sí mismo" y la organización de la vida colectiva. Los actores elaboran múltiples categorías en diferentes contextos de experiencia, que coexisten y no están necesariamente coordinadas de forma racional y que, a su vez, los conectan con otros actores ${ }^{(21)}$.

En dos escritos previos, analizamos y discutimos tres contextos de la experiencia de familiares y amigos de jóvenes, muertos en situación de violencia interpersonal con $\operatorname{pares}^{(22,23)}$ : a) el esclarecimiento de los motivos de la muerte del joven, así como una búsqueda de establecer rupturas y reordenamientos en los lazos sociales de la familia del agresor, y de los jóvenes amigos; b) los pensamientos y las categorías morales que disponen y utilizan los actores para establecer argumentos frente a la muerte del joven, lo que configura la construcción de una reputación moral y social del muerto ${ }^{(24)}$, en la que se destaca un conjunto de repertorios culturales que utilizan los actores para ello; c) un proceso de desintegración del yo, el dolor concomitante y una experiencia de vulnerabilidad corporal que ocasiona la muerte violenta en los actores.

El problema del presente estudio es la profundización de los modos en que estos contextos se articulan, se tensionan o se diferencian en los itinerarios de la experiencia de dichos actores, y los vacíos de sentido que no pueden explicar. Cuando los familiares de los jóvenes muertos en situaciones de violencia interpersonal con otros jóvenes producen una reconstrucción biográfica, y cuando amigos de los muertos reconstruyen su propia vida ¿cómo se articulan los diferentes contextos en la misma experiencia de los actores?; ¿qué problemas enfrentan al vincular el proceso de memoria con las sociabilidades juveniles y la misma sociabilidad familiar?; ¿de qué modo la experiencia de los actores puede metabolizar las relaciones sociales de los jóvenes muertos y cuestionar la naturalización de la violencia entre jóvenes en los contextos sociales barriales? 


\section{METODOLOGÍA}

Se realizó un estudio de casos estudiados en profundidad, de tipo exploratorio y descriptivo, de carácter inductivo, utilizando técnicas de construcción y análisis cualitativo de datos $^{(25)}$. Estas permiten una comprensión del sentido de la acción social, en el contexto de la experiencia personal y desde la perspectiva de los sujetos ${ }^{(26)}$.

Se eligieron dos sujetos de estudio, en poblaciones marginalizadas de Villas de Emergencia de la zona sur de la CABA:

- Familiares de jóvenes muertos entre 15 y 25 años, en situación de violencia interpersonal entre pares, para la reconstrucción de la vida de estos últimos.

- Jóvenes amigos, entre 15 y 25 años, de otros jóvenes muertos en situación de violencia interpersonal entre pares, para la reconstrucción de la vida de los primeros.

Se aplicaron perspectivas del método biográfico y reconstrucción de relatos de vida, ya utilizados en otros estudios previos ${ }^{(27,28)}$. El proceso metodológico implementado comprendió:

a. La aplicación de 4 a 6 entrevistas en profundidad, que incluyeron como tópicos: una caracterización específica de la situación de la muerte del joven; una descripción de los principales espacios de sociabilidad transitados; y una selección de los principales acontecimientos del joven biografiado, los que marcaron "un antes" y "un después" en su vida, así como lo que desencadenó cada uno de ellos.

b. La desgrabación de las entrevistas y la entrega progresiva a los sujetos estudiados de una copia de este material para su lectura y realización de observaciones y comentarios.

c. El ordenamiento de los acontecimientos biográficos por parte de los mismos sujetos de estudio, según sus propios criterios de importancia, a lo largo del proceso de toma de entrevistas. d. La confección de un relato de vida, compuesto por una presentación del joven biografiado (el joven muerto o el amigo del joven muerto) y un listado de los acontecimientos biográficos según el orden de importancia establecido por los propios sujetos.

El grupo de estudio quedó conformado por seis familias de jóvenes varones muertos de 15 a 25 años, y 8 jóvenes varones, de 15 a 25 años, amigos de pares muertos, de sexo masculino.

Se realizó el trabajo de campo para acceder a los casos en dos "villas de emergencia" de la zona sur de la CABA, a partir de la inserción del investigador responsable de este proyecto en un servicio de salud del primer nivel de atención. La selección y el trabajo con la población de estudio fue realizada entre julio de 2014 y julio de 2016.

Para el acceso territorial a los sujetos se utilizaron múltiples vías de acercamiento: referentes comunitarios (principalmente de comedores comunitarios); instituciones que trabajan con población juvenil en los barrios; familiares de jóvenes muertos, a través de personas conocidas por su atención en el servicio de salud en el que se desempeña el investigador responsable del proyecto; jóvenes que tienen relación con este servicio y el profesional responsable del proyecto.

El diseño de estudio y el consentimiento informado elaborado para la participación de los sujetos fueron evaluados por las autoridades del Ministerio de Salud del Gobierno de la Ciudad de Buenos Aires.

Para la toma de las entrevistas se elaboró una guía para cada población de estudio. La recolección de la información fue audiograbada, previo consentimiento informado de los participantes. Para el análisis de los datos, las entrevistas fueron desgrabadas, y se elaboró un sistema de codificación cualitativa para el ingreso de la información de cada uno de los sujetos de estudio. Se procedió al ingreso de la información primaria y de los códigos al software Atlas Ti. A continuación, se realizó el trabajo de codificación del material primario. Finalmente, se procedió 
al análisis por código y a la comparación de estos entre sí; identificando las principales categorías emergentes.

\section{LAS SOCIABILIDADES DESPUÉS DE LAS MUERTES, EL RESENTIMIENTO $Y$ LA VENGANZA}

En el momento inmediato posterior a las muertes, se verifica una tensión y mixtura entre tres contextos de sentidos. Primero, el esclarecimiento de los motivos de la muerte y el reordenamiento de las relaciones sociales. También, la activación del resentimiento que provoca el dolor en el cuerpo, con el surgimiento de un deseo de venganza y eliminación del agresor y sus allegados. Finalmente, aparecen una serie de pensamientos y valores, para efectivizar dicho deseo. Se trata de la construcción de una reputación negativa del agresor y sus allegados.

Las principales circunstancias con las que los actores caracterizan las muertes son:

a. Enfrentamientos entre grupos de jóvenes de diferentes sectores de un mismo barrio o entre grupos de diferentes barrios, en el que se buscan sustraer bienes materiales, producto de los robos, y que también pueden incluir la disputa por relaciones amorosas con mujeres. Las disputas se inscriben en un control territorial de poder y prestigio simbólico que adquieren los objetos materiales. Se pueden incluir aquí, venganzas a víctimas que no forman parte activa de un grupo de sector, sino que pueden tener una adscripción relativa vinculada a la circulación y contacto con un grupo constituido.

b. La muerte es resultado de una venganza por robar en el mismo barrio a otros jóvenes o a vecinos. Es lo que los jóvenes denominan rastrear. Puede tratarse de grupos o de jóvenes que están solos. En la mayoría de los casos, esto está relacionado con obtener recursos para la compra de droga.

c. Los robos violentos entre jóvenes en forma individual, que pueden incluir a dos agresores y que terminan en una muerte. Puede tratarse de situaciones dentro o fuera del barrio. d. Situaciones de muertes que están vinculadas al tráfico drogas en el mismo barrio. Se destacan dos situaciones: un ajuste de cuentas de un narcotraficante, el denominado tranza, a otro joven que le debía plata; o cuando los jóvenes mueren por una venganza por haber robado a un cliente de algún tranza.

Cuando los familiares buscan determinar estas circunstancias, esto se relaciona con el tipo de vínculo de ellos con las sociabilidades juveniles. En la mayoría de los casos, el joven residía o mantenía vínculo cercano con sus familiares de origen, quienes conocían la inserción en su grupo de amigos y podían establecer las situaciones que condujeron a la muerte del joven, e incluso, preanunciarla, advirtiéndole sobre la necesidad de protección. De modo diferente, en algunos casos, los familiares desconocían la inserción social del joven, porque ya no residía con estos y, por ello no podían establecer las causas de la muerte. Esto establece un vacío de sentido, que genera una experiencia de incertidumbre en los vínculos con los vecinos, y con respecto a una inscripción psíquica y social de la muerte.

Junto al resentimiento que busca la eliminación del otro, la experiencia corporal del dolor, puede devenir, contrariamente, una experiencia de vulnerabilidad ante la posible agresión a los propios familiares. Por ello, se produce un proceso de aislamiento y restricción en los vínculos sociales, en la circulación barrial. El deseo de eliminación de los agresores se puede transformar en una experiencia paradojal, ya que a menudo forman parte de un mismo contexto de sociabilidad barrial en el que, incluso, las familias de ambos lados tenían relaciones previas. De ello, resulta una experiencia que atraviesa un momento de máxima vulnerabilidad corporal, pero que no tiene otra opción que el resentimiento. Los cuerpos de los familiares no tienen posibilidades establecer distancia con los agresores y sus allegados, y son obligados a la convivencia con estos, en la circulación cotidiana por el barrio.

Pero los familiares, también, son los destinatarios de la mirada social de los vecinos 
que juzgan las trayectorias sociales juveniles vinculadas a la violencia, el delito y el consumo de sustancias, con argumentos que legitiman la eliminación y el olvido de estos jóvenes en la comunidad barrial. Los jóvenes se pueden constituir en el enemigo interno en el que se podría depositar todo lo moralmente cuestionable de una comunidad lo$\mathrm{cal}^{(29)}$. Cotidianamente, las muertes de estos jóvenes pueden considerarse muertes merecidas ${ }^{(24)}$, un "asunto privado entre jóvenes que no tiene importancia social" ${ }^{\prime(30)}$; y sus allegados se convierten en una larga serie de casos de personas que sufren y experimentan dolor privadamente. Las categorías morales de la mirada social obstaculizan, entonces, la visibilidad pública de las pérdidas y el sufrimiento de los allegados a los muertos. Pero, de cualquier modo, la experiencia de los familiares, se ve interpelada por esos juicios sociales y tienen que exhibir una presentación pública frente a la interpelación social. ¿de qué recursos culturales y materiales disponen los familiares para construir una reputación social del joven muerto, de sí mismos y de sus vínculos con los jóvenes, que pueda diferenciarse de la apelación a la violencia como recurso disponible privilegiado? Las posibilidades de enunciación de una reputación moral del muerto y de la misma familia, en principio, está vinculada a dos dimensiones coexistentes. En primer lugar, se trata de una cuestión política, anclada en el poder material y simbólico de cada familia. Allí, la relación con las instituciones y organizaciones sociales que tuvieron los jóvenes muertos y que poseen las mismas familias se torna fundamental para adquirir una visibilidad social de las muertes. Pero, también las posibles afinidades de las familias con la red del delito, ya sea el robo o comercialización de drogas, pueden constituirse en causales del mantenimiento de la invisibilidad social de las muertes. En segundo lugar, los familiares pueden argumentar un conflicto de poder intergeneracional con los jóvenes en torno a la autoridad, en varios aspectos. Puede tratarse de lo que se ha conceptualizado como "brecha creciente entre consumo simbólico y consumo material", de bienes y servicios por parte de los jóvenes ${ }^{(31)}$. Estos demandan a sus familias objetos de consumo, y estas no pueden responder a esas demandas. Por ello, los jóvenes cuestionan la autoridad familiar y se autonomizan en las sociabilidades juveniles vinculadas al delito, para satisfacer dichas demandas. Los integrantes de las familias pueden destacar sus diferencias con ellos, vinculadas a una ética del trabajo y la educación, e incluso en muchos casos, efectivizar en sus trayectorias estas expectativas. También, el conflicto intergeneracional puede expresarse en una acentuación del conflicto de poder entre la sociabilidad familiar para procurar mantener al joven allí, y las sociabilidades juveniles. Aquí cobra importancia, además, el poder autónomo de los jóvenes vinculado al acceso y disponibilidad de armas de fuego ${ }^{(32)}$.

Se analizó, previamente, el proceso de socialización y las trayectorias de los jóvenes que conducen a la formación de sociabilidades vinculadas al ejercicio de la violencia, el delito y el consumo de sustancias ${ }^{(23)}$. Un conjunto de categorías emergentes permite explicar el funcionamiento de los grupos juveniles. En primer lugar, se trata de la obtención de reconocimiento identitario, en tanto demanda de respeto, que se logra con el establecimiento de una relación violenta y desigual con "otros", y una relación de reconocimiento de demanda de igualdad, con los "mismos", de la propia sociabilidad. Este respeto se obtiene mediante dos categorías fundamentales para los actores: una, es obtener plata fácil, fundamentalmente, mediante el robo, pero también, a través de la participación en las redes de comercialización de drogas; la otra, es el joven ganado, que remite a la atracción que ejerce en las sociabilidades juveniles la figura del joven que obtiene éxito en la actividad del robo y que actúa como una aspiración a la que cada uno de ellos busca acceder.

La muerte de los amigos, cuando los jóvenes compartían una sociabilidad con el fallecido, introduce fisuras, debates, ambivalencias y contradicciones en sus experiencias; las que ponen en cuestión la relación de dichas categorías con la acción del actor. En lugar de un afianzamiento de una cultura 
del pibe chorro, en tanto traducción en acciones de creencias culturales, que garantizarían la reproducción social del delito y la violencia $^{(33)}$, las relaciones sociales y las motivaciones culturales y subjetivas del actor se distancian y fragmentan.

En el momento inmediato a la muerte, el conjunto de categorías que incluye obtención de respeto y plata fácil, y aspirar a ser un joven ganado, sufre un profundo clivaje entre dos dimensiones que entran en tensión entre sí, basado en la competencia entre jóvenes. Por un lado, el reforzamiento de la violencia mediante la ideación de venganza de la muerte frente al grupo agresor $y$, en algunos casos, su efectivización. Allí opera la lógica del restablecimiento del respeto cuestionado entre los jóvenes, basado en la desigualdad material y simbólica; donde la vulnerabilidad y el dolor se convierten en resentimiento. En una segunda dirección, hacia el interior de los grupos, los jóvenes accionan un cuestionamiento del reconocimiento basado en la igualdad. La competencia basada en el consumo individual, revela una desconfianza hacia los pares, a los que se suponía iguales. El compañero es objeto y sujeto de envidia. La "desconfianza hacia los envidiosos" se revela una experiencia de vulnerabilidad y remite al resentimiento del dolor. Los que serían los amigos pueden dejarte tirado o dejarte morir. La lógica de la desigualdad se instala en un grupo que se consideraba de iguales, bajo la premisa de que "todos quieren ser líderes, sentirse más que los demás. Todos quieren ser un grupo". Así, la dimensión cultural del consumo que acentúa el proceso de individuación crea una fisión con el proceso de socialización en el grupo ${ }^{(34)}$. Se observa, además, que este debate está relacionado con diferencias en las perspectivas generacionales juveniles en una misma sociabilidad. A menudo, los jóvenes de más de veinte años son críticos con las expectativas de consumo y los valores de los más chicos, de trece o catorce años, quienes están ingresando en el mismo grupo.

En el contexto de esta escisión de la categoría de obtención de respeto y la visibilización de la competencia entre jóvenes, la experiencia del actor inaugura un proceso de vulnerabilidad en el propio cuerpo y en los vínculos sociales. La figura del resentimiento direccionada hacia los agresores y hacia los mismos pares del grupo, se encuentra, también, con la angustia y la posibilidad de la propia muerte en la experiencia corporal. Es lo que se designa cajeteo, significado como dudar, o tener miedo de volver a ejecutar acciones violentas. Así, el actor reflexiona ellos desperdiciaron la vida y vos tenés que pensar lo que querés para tu vida, cuidarte de robar, poniendo en relación las trayectorias sociales del muerto y de sí mismo con las sociabilidades de las que participan. Este momento coincide con un apaciguamiento de las acciones violentas.

El proceso de vulnerabilidad desencadenado por la muerte y la desconfianza de los otros, comprende un reordenamiento de las sociabilidades y las posiciones sociales que adopta el actor, que puede llevar a un distanciamiento del grupo y conducir a la inclusión en otras sociabilidades distintas; andar solo, con la intensificación del consumo de drogas y la comisión de delitos o, contrariamente, como forma de evitar conflictos; la disolución o reagrupamiento de los jóvenes vinculados al encarcelamiento de pares; la búsqueda del control del robo adentro del mismo barrio, el rastreo, mediante una crítica y disputa moral entre los que roban "adentro" y "afuera" de la misma comunidad. Finalmente, la vulnerabilidad tras la muerte está vinculada a una disputa política, donde interfieren el poder del narcotráfico y el de los jóvenes que roban. El robo puede ser cuestionado por el poder del narco o por los intercambios directos o indirectos entre jóvenes, e incluso, las familias, que trafican y que roban.

\section{LOS LÍMITES DE LAS CATEGORÍAS MORALES Y LOS PENSAMIENTOS: LAS CRÍTICAS DE LOS ACTORES}

Las jerarquizaciones morales de las muertes son un problema de biopolítica y de una distribución desigual de las vulnerabilidades en 
los territorios, que no ha sido consideradas por la bibliografía sobre las muertes violen$\operatorname{tas}^{(35)}$. Zaffaroni ha denominado genocidio por goteo al hecho de dejar que los jóvenes en las poblaciones urbanas marginalizadas de América Latina se maten entre sí y que esas muertes permanezcan invisibles socialmente $^{(36)}$. Mbembe ${ }^{(37)}$ desarrolla un concepto de soberanía definido por la "capacidad para definir quién tiene importancia y quién no la tiene, quién está desprovisto de valor y puede ser fácilmente sustituible y quién, no". Esta soberanía puede ser delegada por el Estado en grupos comunitarios de un territorio, con los cuales puede entrar en intercambios directos o indirectos.

Tanto los familiares, como los jóvenes estudiados, insisten con un guion anticipado de muertes anunciadas: vos sabes que si robás o andás en la tranza, podés terminar muerto. En las reconstrucciones biográficas de las trayectorias de vida de los jóvenes, los familiares moralizan un conjunto de hechos, los que conducen a la muerte y que la efectivizan: el consumo de drogas en un grupo de pares en "la esquina"; la participación en actividades de robo con otros jóvenes y obtener plata fácil; el distanciamiento del joven de su grupo familiar; no escuchar los consejos de los miembros de familia y de otros allegados, sentirse más grande de lo que se es y buscar decidir por sí mismo, el considerar a la escuela como aburrida y de giles. Esta última categoría alude una descalificación peyorativa de los jóvenes.

Familiares y jóvenes interiorizan un pensamiento social de eliminación de los jóvenes, aun cuando los valores mencionados entran en debate en la experiencia del actor. Se intenta racionalizar la muerte de los jóvenes, pero los actores se encuentran permanentemente con el dolor y la interpelación de los muertos, los que hablan e interactúan con sus allegados en los testimonios.

Existe una serie de cuestionamientos de diferentes categorías morales y pensamientos que conducen a un límite impensado en la experiencia de los actores. El debate que se instala en los comienzos de las entrevistas, tanto en familiares, como en los jóvenes, es sobre el concepto de amistad que median los vínculos entre jóvenes. Se presenta al modo de una urgencia que necesita una respuesta. Para los familiares, el cuestionamiento adquiere sentido, en primer término, en una disputa con la sociabilidad del grupo de pares de los muertos: dicen que son tus amigos, pero ¿a qué te condujeron?. Son las malas juntas, un sociologismo de las influencias sociales que arruinaría un supuesto ideal de la sociabilidad familiar, que nunca se pude efectivizar. Pero en un segundo movimiento, los familiares transforman el cuestionamiento en una crítica social que, al mismo tiempo, los involucra y los distancia. Es el consumo como mediador exclusivo de los vínculos en las sociabilidades juveniles, solo sos amigo si tenés algo, los amigos te dejan tirado, no están cuando los jóvenes mueren, y ellos no los recuerdan. El cuestionamiento también los involucra en la formulación de conflictos intergeneracionales, en los que la autoridad de los adultos de la familia es también mediada por el consumo que, al no poder dar una respuesta, quedan en un impase de sentido. Se trata de otra experiencia paradojal: realizan una crítica social del consumo que constituye a las sociabilidades juveniles, pero ellos mismos se sienten en falta para responder a esto y no pueden ejercer una autoridad que le ponga límites a las demandas de consumo juveniles.

Pero, para los familiares, esta discusión sobre el consumo también instala un debate vinculado a las trayectorias de consumos y adicción a las drogas de los jóvenes muertos que conduce, además, a ejercer reclamos por la gran disponibilidad de sustancias y el poder del narcotráfico que opera con impunidad en el proceso de socialización de los jóvenes, y ante el cual ellos se revelan impotentes.

Al profundizar en el análisis que realizan los jóvenes sobre los orígenes y las causas de la competencia entre pares por bienes materiales y simbólicos, que conducirían al ejercicio de violencia y la muerte, ellos aluden a una acumulación de broncas en su experiencia. Es la categoría juntar broncas, la que provocaría un dolor convertido en resentimiento 
al momento de ejercer el acto de agresión y alude a la convergencia de un conjunto de interacciones. En primer lugar, se mencionan diferentes situaciones en la propia familia que provocan dolor al joven. En particular, se destacan haber sido testigo de violencia de género, haber sido objeto de violencia física y emocional o de situaciones de abandono. En segundo lugar, se señala la participación en injusticias cotidianas en el espacio público que incluyen, fundamentalmente, la discriminación para acceder al mercado laboral, por el hecho de provenir de un barrio estigmatizado como "villa", y los enfrentamientos y violencias protagonizados por los jóvenes con las fuerzas de seguridad. Finalmente, se menciona la relación entre pares, donde el joven se da cuenta que no existe la amistad. Son las categorías ya mencionadas de dejarlo tirado, o dejarlo morir.

De este modo, la violencia y la muerte, como imposición de un reconocimiento desigual entre jóvenes, podría pensarse como un problema de historización no resuelta, de reconocimiento del joven y su vinculación con el dolor en múltiples sociabilidades e interacciones, que exceden el enfrentamiento entre pares. Pero, al mismo tiempo, esa acumulación de sucesos y resentimientos se efectivizarían en el acto violento entre jóvenes. Algunas perspectivas han propuesto ubicar el análisis de las violencias en los contextos situacionales más que en la conciencia y la autonomía de las motivaciones del actor; sugiriendo, además, enfatizar el análisis del proceso de "encadenamiento" de diferentes contextos situacionales y "derrame" de las violencias de los actores, en un territorio específico $^{(38)}$. El problema que plantea la experiencia de los jóvenes de nuestro estudio, no es solo el de un encadenamiento y derrame de violencias de diferentes contextos de interacción. Se trata de los posibles modos de desanudamiento de los otros contextos, a partir de los enfrentamientos específicos entre jóvenes y, también, las posibles formas de distribución del afecto del resentimiento hacia otras interacciones; así como las posibles transformaciones del mismo en otra forma de dolor.

\section{DISCUSIÓN Y CONCLUSIONES: LA EXPERIENCIA PARADOJAL DE LOS ACTORES Y EL IMPASE DEL DOLOR}

Del recorrido del presente estudio surge una compleja experiencia de los actores tras la muerte de un joven. El trabajo que se les impone para lidiar tanto con las relaciones sociales del muerto, en las que están comprometidos de una u otra manera, así como con los distintos pensamientos y categorías con las que intentan construir argumentos, revelan sus límites e impases de sentido. La vulnerabilidad y el dolor que atraviesan la experiencia, por momentos, intenta cerrar sentidos, con la reanudación de la violencia, bajo la figura de la venganza o el restablecimiento de respeto mancillado. Pero, esta experiencia conducida por el resentimiento deja más preguntas y sentidos en la invisibilidad, que los que pretende clausurar.

Para defender este argumento, es posible discutir que no es posible para los actores realizar el trabajo que impone el reordenamiento de las relaciones sociales, así como el de encontrar juicios y pensamientos que expliquen la violencia que desencadena la muerte, si aquéllos no realizan un trabajo de subjetivación que parta de un reconocimiento corporal y social del dolor, y las vulnerabilidades que desencadena.

La bibliografía sociológica, de la antropología de las moralidades y de la criminología cultural con la que se dialogó en este trabajo, permite explicar el proceso de exclusión y segregación territorial, así como la disputa identitaria por el reconocimiento y de valores que encarna la violencia interpersonal y las muertes entre jóvenes. Lo que surge como un problema, que este estudio aporta en su caracterización, son las condiciones de la experiencia de los actores en que el dolor y la recuperación de la memoria de las muertes, les podría permitir realizar una crítica que cuestione las relaciones sociales y juicios comunitarios que legitiman el ejercicio de la violencia y la muerte.

Las perspectivas biográficas de los familiares y de los amigos de los jóvenes muertos permiten constatar una experiencia de los 
actores, en la que se distancian las prescripciones sociales y las "motivaciones individuales", "la cultura y la estructura social se separan" y la identidad "se convierte en una tensión creciente entre identidad para otro y la identidad de sín $i^{\prime(39)}$

En el caso de los familiares, se trata de una experiencia paradojal. Al reconstruir la vida de los jóvenes muertos, deberían resolver un desafío, una prueba social(40). Por un lado, se ven obligados a construir una reputación del joven y de la propia familia con relaciones, conflictos y pensamientos sociales que procuran eliminar a los jóvenes de la comunidad. Y, al mismo tiempo, tendrían que recuperar su memoria en una experiencia personal de dolor y de desintegración del yo. Existiría una divergencia y una paradoja: los familiares deben dar cuenta de su experiencia personal acerca de las relaciones sociales del muerto pero, al dar cuenta de estas relaciones, surge el dolor, y son cuestionados por los juicios morales que comporta el ejercicio de la violencia de los jóvenes. La experiencia del dolor puede legitimar tanto como cuestionar la moral comunitaria que engendra la violencia y la muerte. El actor puede cuestionar personalmente la moral imperante, pero al no existir un reconocimiento social del dolor en el espacio público, las posibilidades de crítica social y de la construcción de otra moral comunitaria que cuestione la violencia y la muerte se encuentran limitadas de enunciarse $^{(41)}$. El análisis de la experiencia revela que son vidas que no tienen "la capacidad de ser lloradas", porque no tienen normas sociales que las reconozcan como precarias y vulnerables, a lo largo de sus trayectorias sociales ${ }^{(42)}$.

En el caso de los jóvenes amigos de pares muertos, es posible formular y caracterizar una experiencia paradojal del reconocimiento. En ella, interactúan, se tensionan y conviven, contradictoriamente, tres dimensiones. En primer lugar, se trata de una demanda de reconocimiento entre jóvenes basado en una desigualdad jerárquica, que impone el ejercicio de la violencia y la muerte como forma naturalizada socialmente ${ }^{(13,11)}$. Aquí, las demandas de reconocimiento son accionadas por grupos o jóvenes en forma individual, y se basan en la obtención de un poder material y simbólico. Esto se produce en condiciones de exclusión y segregación sociales, en la cuales la sobrevivencia cotidiana está sometida a redes de microeconomías ilegales, vinculadas al robo y la comercialización de drogas. Una segunda dimensión, la constituye la demanda de un mutuo reconocimiento, en tanto iguales. Son los insistentes reclamos de amistad, lealtad, compañerismo y no abandono entre jóvenes ante la posibilidad de la muerte. Esto ocurre en la sociabilidad de los grupos, que es cuestionada cuando los jóvenes perciben que el consumo y los intereses individuales priman por sobre los vínculos entre iguales. Finalmente, surge en la experiencia una necesidad de cada joven de reconocimiento del dolor en el propio cuerpo, cuando la pérdida de un amigo es puesta en relación con la propia reconstrucción biográfica, y con las sociabilidades que se compartían con el muerto. Las trayectorias personales pueden devenir trayectorias sociales a través de un ejercicio de la memoria que recorre los grupos de socialización ${ }^{(43)}$. Allí, cada joven se compara con sus amigos muertos, reconociéndose mutuamente en una experiencia de dolor común, en sociabilidades que comparten la violencia y la muerte, lo cual se expresa en la categoría recurrente las muertes que veías afuera, ahora te tocan a vos. Es un proceso de duelo que se inscribe en las biografías como una experiencia de la vulnerabilidad social, constituida por una identificación de los cuerpos de cada uno como vulnerables, en relación con el de los otros ${ }^{(17)}$. Esto puede inaugurar diferentes acciones de cuidado del actor, hacia sí mismo y hacia los otros: él mismo procura distanciarse de las sociabilidades que provocan violencia y muerte e insertarse en otras diferentes. En dirección contraria, la categoría juntar broncas puede convertir el dolor en resentimiento, negándose la vulnerabilidad corporal y exacerbando el ejercicio de la violencia tras una muerte. Así, este resentimiento podría reenviar la experiencia a la demanda de un reconocimiento jerárquico desigual entre jóvenes, buscando con una 
nueva ofensa desterrar la experiencia corporal de inseguridad ${ }^{(17)}$.

Lo que se torna paradojal en la relación entre estas tres dimensiones del reconocimiento, son las distintas posibilidades de un ejercicio de la memoria en sus biografías frente a la naturalización del punto de partida de la experiencia: ellos construyen identidades en función de una otredad de sí mismos, con la cual se enfrentan y se humillan mediante el recurso disponible de la violencia y la muerte, disputándose objetos de consumo material y simbólico. Este recurso les sustrae los orígenes de las trayectorias sociales comunes en que se socializan como iguales.

Para que el impase de sentido de la experiencia de los actores pueda encontrar otros caminos y efectivizar una crítica social más amplia, es necesario que la memoria y el dolor puedan entrar en relación con las interacciones y sociabilidades en el espacio público del presente. Es posible esbozar un itinerario de intervención social territorial con las familias y los jóvenes allegados a los muertos, que parta del dolor y las reconstrucciones biográficas, en el que la experiencia de los actores pueda trasladarse al espacio público de los mismos barrios. Se trataría de dos movimientos: primero, colocar en dicho espacio las imágenes de los recuerdos de los muertos, estableciendo un debate con los juicios comunitarios que procuran la eliminación y olvido de los jóvenes muertos; $y$, segundo, promover la expresión y el hecho de compartir el dolor, en las mismas sociabilidades, y con otros actores sociales e institucionales. En esta dirección, la utilización de la producción e instalación social de imágenes plásticas, fotográficas y audiovisuales, pueden ser útiles para acompañar la experiencia de los actores ${ }^{(44)}$.

\section{AGRADECIMIENTOS}

El presente trabajo forma parte del proyecto de investigación "Caracterización y efectos psicosociales de las muertes violentas de jóvenes en las poblaciones urbanas de extrema pobreza: una perspectiva biográfica en los contextos de las sociabilidades juveniles", 2014-2016, ejecutado desde el Consejo de Investigación en Salud, Dirección General de Docencia e Investigación, Ministerio de Salud del Gobierno de la Ciudad de Buenos Aires, aprobado con registro $N^{\circ} 161$, del año 2014. Se contó con el patrocinio del Instituto Universitario de Ciencias de la Salud, Fundación H. A. Barceló, sede Buenos Aires.

\section{REFERENCIAS BIBLIOGRÁFICAS}

1. Comisión Económica para América Latina. Panorama social de América Latina 2008 [Internet]. Santiago de Chile: Naciones Unidas, CEPAL; 2008 [citado 01 abr 2018]. Disponible en: https://tinyurl. com/yaaubmk4.

2. Spinelli $H$, Alazraqui $M$, Macías G, Zunino G, Nadalich JC. Muertes violentas en la Ciudad Autónoma de Buenos Aires: una mirada desde el sector salud [Internet]. Buenos Aires: CEDES; 2005 [citado 01 abr 2018]. Disponible en: https://tinyurl. com/ycjfd3nm.

3. Briceño León R. La violencia homicida en América Latina. América Latina Hoy. 2008;50:103-116.

4. Corte Suprema de Justicia de la Nación. Homicidios dolosos 2011: Ciudad Autónoma de Buenos Aires. Buenos Aires: Corte Suprema de Justicia de la Nación, Instituto de Investigaciones y de Referencia Extranjera; 2012 [citado 01 abr 2018]. Disponible en: https://tinyurl.com/y7wnrg3v.

5. Chaves M. Jóvenes, territorios y complicidades: una antropología de la juventud urbana. Buenos Aires: Espacio Editorial; 2010.

6. Míguez D, Isla A. Entre la inseguridad y el temor: instantáneas de la sociedad actual. Buenos Aires: Paidós; 2010.

7. Müller C, Hoffmann X, Nuñez R, Vallejos C, Innamoratto MG, Canavessi JJ, Palacio E, Krause M. Inseguridad social, jóvenes vulnerables y delito urbano: experiencia de una política y guía metodológica para la intervención. Buenos Aires: Espacio; 2012. 
8. Pegoraro JS. Notas sobre los jóvenes portadores de violencia juvenil en el marco de las sociedades pos-industriales. Sociologias. 2002;(8):276-317.

9. Segura R. Elementos para una crítica de la noción de residencial socio-económica: desigualdades, desplazamientos e interacciones en la periferia de La Plata. Quid. 2012;16(2):106-133.

10. Rodriguez E. Malvivientes: jóvenes pobres y conflictividades sociales; mitos y realidades en torno al microdelito. En: Federico R, (comp.). Dossier de jóvenes y legalidad: reconfiguraciones en el abordaje de la conflictividad penal juvenil. La Plata: Ediciones de Periodismo y Comunicación, Universidad Nacional de La Plata; 2012.

11. Zubillaga V. Los varones y sus clamores: los sentidos de la demanda de respeto y las lógicas de la violencia entre jóvenes de vida violenta de barrios de Caracas. Espacio Abierto. 2007;16(3):577-608.

12. Honneth A. La lucha por el reconocimiento: por una gramática moral de los conflictos sociales. Barcelona: Crítica; 1997.

13. Riaño Alcalá P. La memoria viva de las muertes: lugares e identidades juveniles en Medellín. Análisis político. 2000;13(41):23-39.

14. Garriga Zucal J, Noel G. Notas para una definición antropológica de la violencia: un debate en curso. Publicar en Antropología y Ciencias Sociales. 2010;VIII(IX):97-120.

15. Balbi F. De leales, desleales y traidores: valor moral y concepción de política en el peronismo. Buenos Aires: Antropofagia; 2007.

16. Noel G. De los códigos a los repertorios: algunos atavismos persistentes acerca de la cultura y una propuesta de reformulación. Revista Latinoamericana de Metodología de las Ciencias Sociales. 2013;3(2):1-30.

17. Butler J. Vida precaria: el poder del duelo y la violencia. Buenos Aires: Paidós; 2006.

18. Villa A. La relación entre pensamiento y memoria y las condiciones de transmisión en Walter Benjamin: notas para reconfiguraciones identitarias juveniles. En: Korinfeld D, Villa A, (comps.). Juventud, memoria y transmisión: pensando junto a Walter Benjamin. Buenos Aires: Noveduc; 2012.

19. Delor F, Hubert M. Revisiting the concept of "vulnerability". Social Science \& Medicine. 2000;50(11):1557-1570.

20. Dubet F. El trabajo de las sociedades. Buenos Aires: Amorrortu editores; 2008.
21. Cefai D. Diez propuestas para el estudio de las movilizaciones colectivas: de la experiencia al compromiso. Revista de Sociología. 2011;(26):137-166.

22. Villa A. "Los pibes tienen muchos berretines": muertes entre jóvenes, contextos de experiencia y reconstrucciones biográficas. En: Di Leo PF, Camarotti AC, (dirs.). Individuación y reconocimiento: experiencias de jóvenes en la sociedad actual. Buenos Aires: Teseo; 2015. p. 237-271.

23. Villa A. La vinculación de las sociabilidades con las biográficas juveniles: una perspectiva desde la experiencia del dolor ante la violencia interpersonal entre jóvenes [Internet]. En: Beretta D, Cozzi E, Estévez MV, Trincheri R, (comps.). Estudios sobre juventudes en Argentina $\mathrm{V}$ : Juventudes en disputa: permeabilidad y tensiones entre investigaciones y políticas. Rosario: RelJA, CONICET, Universidad Nacional de Rosario; 2017 [citado 20 nov 2017]. Disponible en: https:// tinyurl.com/ybyq392n.

24. Bermúdez NV. "Y los muertos no mueren...": una etnografía sobre clasificaciones, valores morales y prácticas en torno a muertes violentas (Córdoba-Argentina). Berlín: Editorial Académica Española; 2011.

25. Sautú R. Todo es teoría: objetivos y métodos de investigación. Buenos Aires: Lumiere; 2003.

26. Vasilachis de Gialdino I, (coord.). Estrategias de investigación cualitativa. Buenos Aires: Gedisa; 2007.

27. Di Leo PF, Camarotti AC, (eds.). "Quiero escribir mi historia": vidas de jóvenes en barrios populares. Buenos Aires: Biblos; 2013.

28. Leclerc-Olive M. Temporalidades de la experiencia: las biografías y sus acontecimientos. Iberofórum-Revista de Ciencias Sociales de la Universidad Iberoamericana. 2009;IV(8):1-39.

29. Reguillo R. Jóvenes y medios: la construcción del enemigo. Chasqui. 1997;(60):16-19.

30. Cozzi E. Dos relatos de la muerte joven [Internet]. Cosecha roja; 16 may 2014 [citado $01 \mathrm{abr}$ 2018]; Disponible en: https://tinyurl.com/ydbtg6ma.

31. Hopenhayn M. La juventud latinoamericana en sus tensiones y sus violencias. En: Moro J, (ed.). Juventudes, violencia y exclusión: desafíos para las políticas públicas. Guatemala: Magna Terra editores; 2005. p. 29-54.

32. Zaluar A. Juventude violenta: processos, retrocesos e novos percursos. Dados. 2012;55(2):327365. 
33. Tonkonoff S. Juventud, exclusión y delito: notas para la reconstrucción de un problema. En: Federico $\mathrm{R}$, (comps.). Dossier de jóvenes y legalidad: reconfiguraciones en el abordaje de la conflictividad penal juvenil. La Plata: Ediciones de Periodismo y Comunicación, Universidad Nacional de La Plata; 2012.

34. Araujo K, Martuccelli D. La individuación y el trabajo de los individuos. Educação e Pesquisa. 2010;36:77-91.

35. Butler J. Can one lead a good life in a bad life? Radical Philosophy. 2012;(176):9-18.

36. Zaffaroni ER. Los Derechos Humanos como programa y realidad. Revista Pensamiento Penal. 2014. Disponible en: https://tinyurl.com/ybrhfm5a.

37. Mbembe A. Sobre el gobierno privado indirecto. En: Necropolítica. Santa Cruz de Tenerife: Melusina; 2011. p. 77-120.

38. Auyero J, Berti MF. La violencia en los márgenes: una maestra y un sociólogo en el conurbano bonaerense. Buenos Aires: Katz; 2013.
39. Dubet F, Martuccelli D. ¿En qué sociedad vivimos? Buenos Aires: Losada; 2001.

40. Martuccelli D, Singly F. Las sociologías del individuo. Santiago de Chile: LOM Editores; 2012.

41. Das V. Sujetos de dolor, agentes de dignidad. Bogotá: Universidad Nacional de Colombia; 2008.

42. Butler J. Marcos de guerra: las vidas Iloradas. Buenos Aires: Paidós; 2010.

43. Halbwachs M. La memoria colectiva. Zaragoza: Prensas Universitarias de Zaragoza; 2004.

44. Villa A, Valado D. Las muertes violentas de jóvenes en la zona sur de CABA: recuperación de la memoria a través de la producción territorial de murales y fotografías. En: X Seminario de Políticas de la Memoria; Arte, memoria y política; 2017; Buenos Aires.

FORMA DE CITAR

Villa AM. Muertes invisibles entre jóvenes en la zona sur de la Ciudad de Buenos Aires: reconstrucciones biográficas e itinerarios de la experiencia de familiares y amigos. Salud Colectiva. 2019;15:e1707. doi: 10.18294/sc.2019.1707.

Recibido: 12 de abril de 2018 | Versión final: 15 de agosto de 2018 | Aprobado: 28 de agosto de 2018

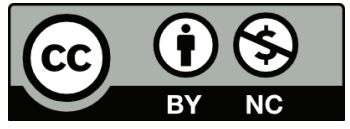

Esta obra está bajo una licencia de Creative Commons Reconocimiento-NoComercial 4.0 Internacional. Reconocimiento - Permite copiar, distribuir y comunicar públicamente la obra. A cambio, se debe reconocer y citar al autor original. No Comercial - Esta obra no puede ser utilizada con finalidades comerciales, a menos que se obtenga el permiso. 